\title{
DEM Simulation to Determine the Influence on the Experimental Results of Tests of Iron Pellets When the Dimensions of the Test Device Are Varied
}

\author{
Yerko Aguilera-Carvajal' ${ }^{\text {, Yonathan Tapia Robledo}{ }^{1}, \text { Sebastián Perez Cortes }}{ }^{2}$ \\ ${ }^{1}$ Universidad de La Serena, La Serena, Chile \\ ${ }^{2}$ Universidad de Santiago, Santiago, Chile \\ Email: yerko.aguilera@userena.cl,ytapiar@gmail.com, sebastian.perez@usach.cl
}

How to cite this paper: Aguilera-Carvajal, Y., Robledo, Y.T. and Cortes, S.P. (2021) DEM Simulation to Determine the Influence on the Experimental Results of Tests of Iron Pellets When the Dimensions of the Test Device Are Varied. International Journal of Modern Nonlinear Theory and Application, 10, 65-80.

https://doi.org/10.4236/ijmnta.2021.102005

Received: April 9, 2021

Accepted: May 22, 2021

Published: May 25, 2021

Copyright $\odot 2021$ by author(s) and Scientific Research Publishing Inc. This work is licensed under the Creative Commons Attribution International License (CC BY 4.0).

http://creativecommons.org/licenses/by/4.0/

\begin{abstract}
The current study is based on the DEM computer simulation of three experimental test devices with different dimensions to determine the difference in the results of the formation of shear and repose angles that the particles experience when grouped under the action of the gravitational force. In this respect, the experimental test devices with different height, width, and depth were geometrically modeled with iron pellet particles using morphology and a granulometric variation from $6 \mathrm{~mm}$ to $9 \mathrm{~mm}$ of equivalent diameter in its spherical shape. Depending on the results obtained, a reliable size of the experimental test device will be available to obtain the necessary data for a correct adjustment of the calibration parameters for the DEM simulation of mining-metallurgical processes that use granulated material of iron pellet.
\end{abstract}

\section{Keywords}

Discrete Elements Method, Calibration, Test Device, DEM Parameters

\section{Introduction}

The growing use of computer simulation through the discrete elements method (DEM) in mining, food, processing and farming industry to characterize and improve their processes has allowed an efficient development of them, allowing in turn to know the impact of their processes on the environment.

The method developed by Cundall, P. A., \& Strack [1] at the beginning of the 70's and its subsequent current evolution [2] [3] have allowed together with the development calculation processes using graphics processors (GPU) [4], a sus- 
tained advance in new methods and multiphase simulation possibilities [5] and a growing number of new publications on the matter in a wide field of sectors such as geomechanics [6], underground mining and open-pit mining [7] [8], CFD-DEM in pyrometallurgical processes [9], agricultural process equipment [10] and significant advances in pharmaceutical industry processes [11].

Given the relevance of benefits associated with the development of DEM Simulations, it is where the calibration of its behavior parameters plays a fundamental role to generate simulations that adjust to reality. The importance of calibrations lies in the fact that the results of the simulations must be developed under the physical phenomena associated with the displacement dynamics of the particles and the interaction between them [12], due to this, it is necessary to be able to count on the characterization of the test devices to obtain reliable experimental parameters that will be used for the configuration of the adhesive model in computer simulations using the discrete elements method.

The fundamental concept of the discrete element method is based on the interaction of granular materials through a finite number of elements called particles which interact with each other when they come into contact. The interaction between them is defined by the contact models. Each contact model has a variety of different parameters, which must be assigned numerical values. These parameters, as well as other properties of the particles, influence the macroscopic behavior of the simulated material. Since there is no computer approach to determine numerical parameter values directly from a system response, the models must be calibrated. Calibration is an iterative process, which tries to identify a set of parameters whose resulting response from the system corresponds as closely as possible to the desired one. Due to the long calculation times of the DEM simulation, the number of calculations required should be kept as low as possible.

The iterative process of searching for adequate parameter values is highly inefficient and in some cases very difficult to find, so the experimental obtaining of the calibration values is a more efficient method and with high probability than the values entered into the models of adhesion give results adjusted to the phenomena of actual particle flow. In this sense, progress in the improvement of calibration processes has been developed by different researches [3] [13] but as indicated, the main disadvantage of iteration methods is the extensive computational time required, and in some cases can reach thousands of iterations before finding calibration values satisfactory as stated [14] in the research of a methodical calibration process for discrete element models

The experiences carried out in accordance with establishing the variations of the results of the calibration tests in experimental test devices have shown that the dimensions of the test devices vary according to the different length scales used as indicated by the experience of [15].

Although advances in calibration methods have evolved notably, such as the use of artificial intelligence exposed by [16] based on genetic algorithms as well as other more advanced techniques in the use of these advanced calculation tools 
exposed by [17] on models of surface response and neural networks such as the work carried out by [18], they require necessarily a satisfactory tuning and iterative search for calibration values in addition to the coupling of other external numerical simulation software.

From what has been said about the iterative techniques and based on the scarce development in the search for experimental validation methodologies, the current research focuses on the differences that occur when using parameter determination devices for DEM simulation in a simple way and with a high percentage of accuracy, which allows substantial savings in the calculation processes in search of satisfactory results for the calibration of simulation models. To obtain a comparative model and obtain their differences, three devices of different dimensions were geometrically modeled with spherical particles whose granulometry varied from $6 \mathrm{~mm}$ to $9 \mathrm{~mm}$ under identical material input conditions and simulation time.

This research work methodically establishes the variables that must be considered for the construction of a device model that allows results of experimentation for calibration of reliable DEM parameters of granular material in iron pellets, avoiding costly time to perform calibrations with the iterative method in addition to indicating which are the variables that generate significant differences in the results of the tests.

\section{Formulation of the Discrete Element Method}

The basic foundations of the discrete elements method are based on the fact that the particles basically describe two types of movements, the first is translational and the second is rotational, these happen when the interaction between them occurs and when a particle interacts with the walls of the system that contains it and/or with a circulating fluid in the means of analysis. When the movement of the particles occurs, an exchange of moment and energy is generated. Through the interactions that occur between particles it can determine the resulting force on it [19].

This approach allows using Newton's second law of motion to obtain the motion of individual particles, assuming that the system contains $n$ discrete elements, the particles $i$ have a mass $\left(m_{i}\right)$ and a moment of inertia $\left(l_{i}\right)$, the equations for the translational and rotational motion can be written as:

$$
\begin{gathered}
m_{i} \frac{\mathrm{d} v_{i}}{\mathrm{~d} t}=\sum_{j} F_{i j}^{c}+\sum_{k} F_{i k}^{n c}+F_{i}^{f}+F_{i}^{g} \\
l_{i} \frac{\mathrm{d} w_{i}}{\mathrm{~d} t}=\sum_{j} M_{i j}
\end{gathered}
$$

where $v_{i}$ correspond to the speed of translation, while $w_{i}$ is the component of the angular velocity of the particle $i$, the contact force and torque exerted by a particle $j$ or walls on the particle $i$ are represented as $F^{c}, M_{i j}$ respectively, $F^{c}, M_{i j}$ is the force of $F, M_{i j}$ non-contact caused by particle $k$ on particle $i$, while $f$ is a component of the force produced by the particle-fluid interaction on particle $i$ 
and $F^{y}$ in the gravitational force. In Figure 1 the forces and pairs involved in a DEM simulation [20] are shown schematically.

\subsection{Contact Forces between Particles}

The contact between two particles generally does not occur in a single point, due to the geometric deformation that the contact zone possesses is defined by a finite area, which is equivalent to the contact that occurs between two rigid bodies that slightly overlap in the DEM. In Figure 2 the contact force distribution over an area can be decomposed into two forces, one normal $\left(F_{n}\right)$ and the other tangential $\left(F_{t}\right)[21]$.

$$
F=F_{n}+F_{t}=f_{n} n+F_{t}
$$

The normal unit vector $(n)$ that allows the union of the center of the particles with a direction outside the particle $i$. Using a constitute model it is possible to obtain the contact between spherical particles shown in Figure 3.

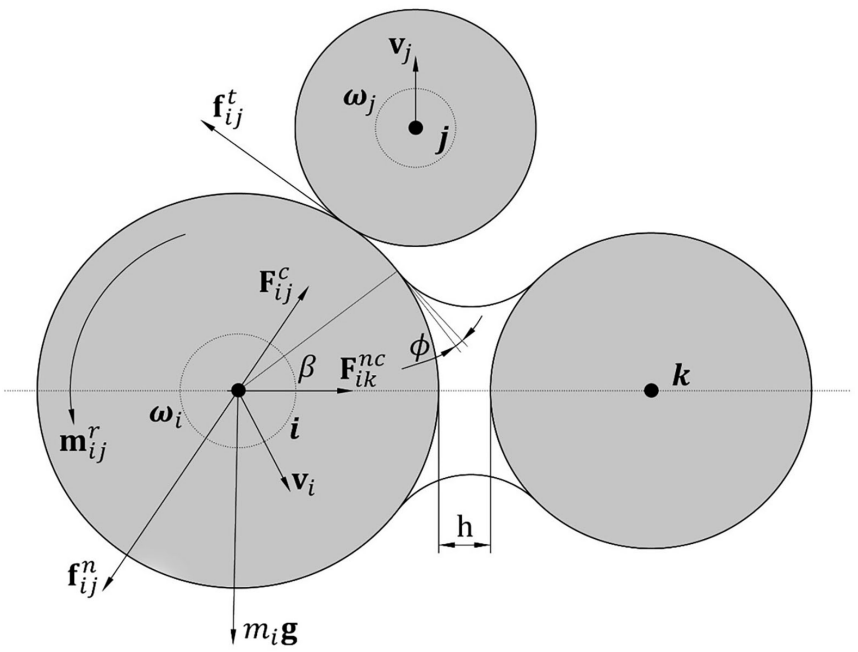

Figure 1. Forces and pairs involved in DEM Simulation.

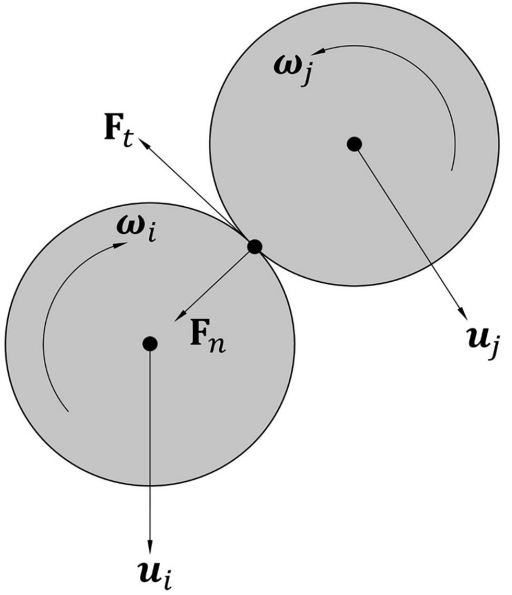

Figure 2. Decomposition of the contact force into its normal and tangential components. 


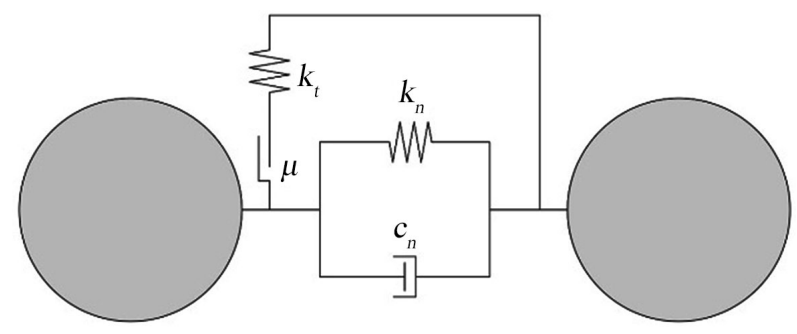

Figure 3. DEM contact interface.

At the contact interface $k_{n}$ and $k_{t}$ are the normal and tangential stiffness respectively, $k_{n}$ the friction coefficient and $C_{n}$ the contact damping coefficient.

The normal component of the norm force is the result of the sum of the elastic normal force $\left(f_{n e}\right)$ and the damping force $\left(f_{n d}\right)$.

$$
f_{n}=f_{n e}+f_{n d}
$$

\subsubsection{Elastic Normal Contac Force}

The elastic normal contact force $\left(f_{n e}\right)$ is proportional to the value of the normal stiffness $\left(k_{n}\right)$ and to that of the penetration of the particle surfaces $\left(u_{r n}\right)$.

$$
f_{n e}=k_{n} u_{r n}=k_{n}\left(d-r_{i}-r_{j}\right)
$$

where $d$ corresponds to the distance between centers that is generated when contact between particles occurs, $r_{i}$ and $r_{j}$ is the radius of the particle $i, j$ respectively. For the case studies that the particles do not have cohesion with each other, there will be no normal elastic contact force (or tension). What causes that when $u_{r n} \leq 0$ the value of the normal tension force $f_{n e}=0$ is set as null.

\subsubsection{Normal Damping Contact Force}

The normal damping contact force $\left(f_{n d}\right)$ is required to cause a decrease in the oscillations of the contact forces, in addition to dissipating the kinetic energy of the particles. For this type of force it must be assumed that it is viscous [22].

$$
f_{n d}=c_{n} v_{r n}
$$

$V_{r n}$ is the relative velocity of the normal component between the centers of the particles.

$$
v_{r n}=\left(u_{j}-u_{i}\right) n
$$

Considering that the damping coefficient $\left(c_{n}\right)$ is contained within the critical damping $\left(c_{c r}\right)$ [23], we have:

$$
C_{c r}=\sqrt{\frac{m_{i} m_{j} k_{n}}{m_{i}+m_{j}}}
$$

\section{DEM Calibration}

The calibration in the discrete elements method is based on obtaining optimal parameters at the time that an interaction between solid bulk particles occurs, which are entered as input values in the simulation of any process for obtaining 
solid material at in bulk, the data sought to be obtained by means of the calibration are the static and dynamic friction coefficients, coefficient of restitution and rolling coefficient [24].

\subsection{Calibration by Angle of Repose Test}

Obtaining the angle of repose consists in determining the angle of inclination with respect to the horizontal when solid bulk material of different dimensions is stacked, obtaining this value depends on the method used in experimental tests or computer simulation, and there are four ways to obtain this result [25].

The techniques of calibration by testing the angle of repose are pouring, draining and dynamic repose.

\subsection{Calibration by Inclined Plane Test}

The inclined plane test is used to determine the coefficients of sliding and rolling friction between the particle and a flat surface of different material. For the determination of the coefficient of sliding friction, a grain of material having a flat side is selected. The granular particle is placed on a horizontal flat wall, to keep the granulated material in a stationary state and thus avoid a possible rolling. This approach ensures that the particle always slides before it starts rolling so that only the slip component can be measured. Then gradually the plane is inclined until the particle begins to slide [26].

$$
\mu=\tan (\varnothing)
$$

\subsection{Calibration by Coefficient of Restitution Test}

This test is used to measure the coefficient of restitution of the particle (e), by means of the ratio between the relative velocities of the particle after and before the impact, when spherical particle of the granulated material is dropped on a horizontal surface. To facilitate obtaining the coefficient, the ratio of the square root of the rebound and the height of fall is usually used, and not the velocity ratio. The physically measured value of the coefficient of restitution is directly used in the DEM model so that the particle in the method has the same dissipation energy property as the real particle [27].

\section{Results and Discussion}

The design of experimentation (DOE) of this research work was based on the series of tests to determine the variations of the results against different dimensions of the same test device design to obtain DEM calibration parameters, for the simulation of the calibration process the Rocky DEM software was used to generate the virtualized tests of the tests of different devices. The material used is based on the properties and morphology of mineral iron pellets, where its main values are shown in Table 1.

For the experimentation and selection according to the DOE, the braking technique was selected for the determination of the angle of repose, which basi- 
cally consists of performing the test with a box-shaped device with a horizontal separation located in the middle distance of the vertical plane, which has a gate and an opening in the upper part where the solid bulk material is deposited as shown in Figure 4. The test process consists of filling the upper part of the device with particles, when the filling is finished, the opening of the gate that has the central division is configured, which causes a large part of the granular material to pass from the upper part of the device to the lower part, due to the effect of gravity, which begins to stack since the lower horizontal cover prohibits its displacement, with this an angle is generated between the lower cover and the material deposited on it, called the angle of repose, while the remaining material remains stored in the upper part since it fails to break its stationary state, it generates an angle with central division, this take the name shear angle.

Table 1. Parámeters iron ore pellets.

\begin{tabular}{|c|c|}
\hline \multicolumn{2}{|c|}{ Iron ore pellet } \\
\hline Density & $3700\left(\mathrm{~kg} / \mathrm{m}^{3}\right)^{\mathrm{a}}$ \\
\hline Particle density & $2200\left(\mathrm{~kg} / \mathrm{m}^{3}\right)^{\mathrm{a}}$ \\
\hline Young's modulus & $70(\mathrm{Gpa})^{\mathrm{b}}$ \\
\hline Poisson ratio & $0.2^{\mathrm{b}}$ \\
\hline \multicolumn{2}{|c|}{ Friction coefficient } \\
\hline Static friction & $0.6^{c}$ \\
\hline Dynamic friction & $0.45^{\mathrm{d}}$ \\
\hline Rolling friction & $0.3^{\mathrm{c}}$ \\
\hline Restitution coeficient & $0.45^{c}$ \\
\hline
\end{tabular}

These values were obtained from: ${ }^{\mathrm{a}}$ [13] (Gustafsson et al., 2013). ${ }^{\mathrm{b}}$ [3] (Gustafsson et al., 2013). ${ }^{\mathrm{c}}$ [28] (Chen et al., 2018). ${ }^{\mathrm{d}}$ [29] (Schott et al., 2016).

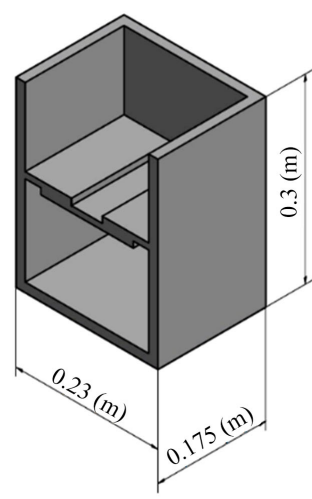

Device 1

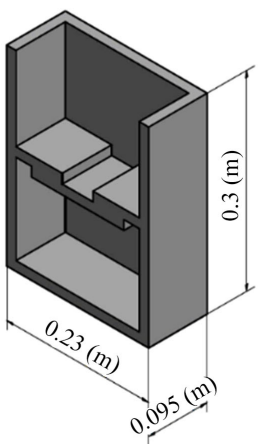

Device 1

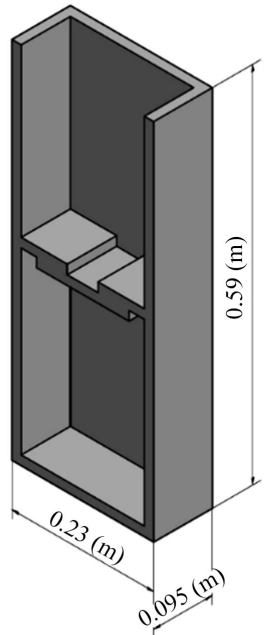

Device 3

Figure 4. Test device. 
Figure 5 shows a calibration by obtaining the angle of repose by means of drainage technique, in the upper part the shear angles are observed with respect to the horizontal, while in the lower part the angles of repose generated when solid material is stacked.

In the discrete elements method, spherical shaped particles are used [30], as can be seen in Figure 6, for its approach in this study there are particles whose dimensions are within the range of 3 to $6.6 \mathrm{~mm}$, at in turn, there will be 3 test devices similar to Figure 4, each with variations in their measurements.

The shape and main dimensions of the iron pellet particles are shown in Figure 7, where from the dimensions in accordance with the granulometry obtained, the equivalent volumes of the particles in their spherical shape were specified.

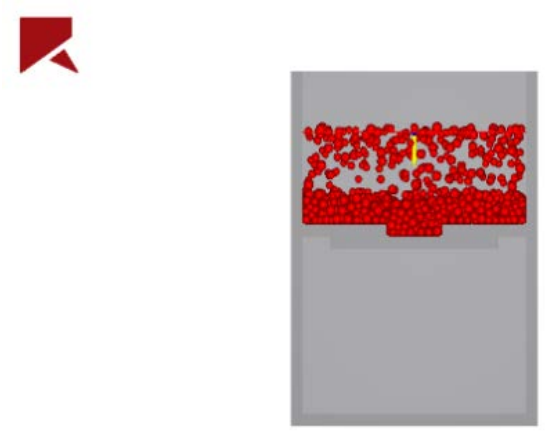

Time 1: Material fill

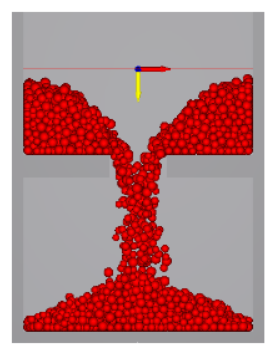

Time 3: Material collection

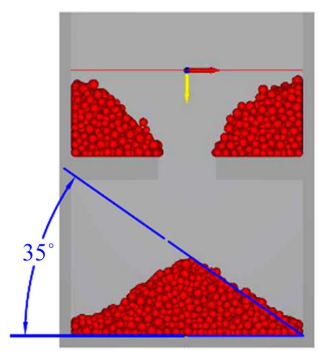

Angle of repose

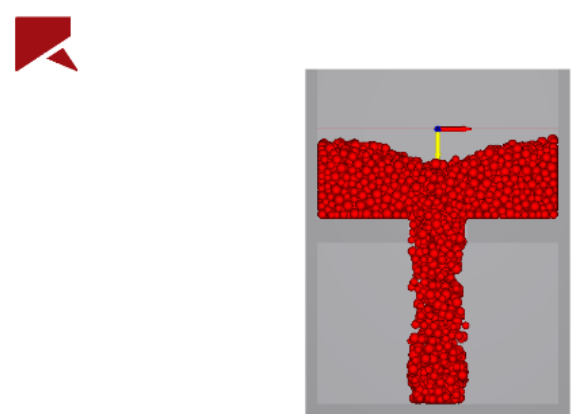

Time 2: Material download
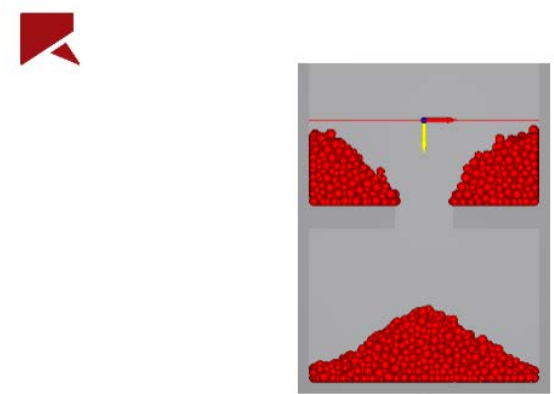

Time 4: Material repose
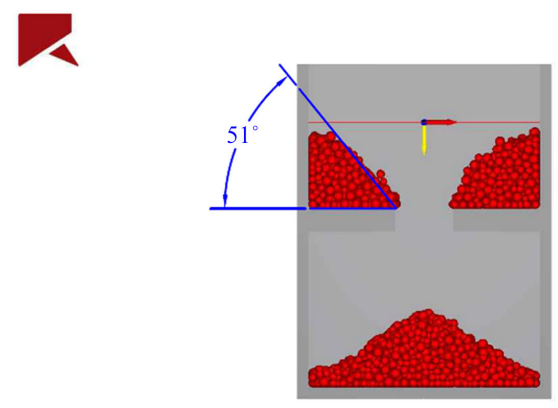

Drawdown angle

Figure 5. Process of formation of the repose and drawdown angle using the drainage technique. 


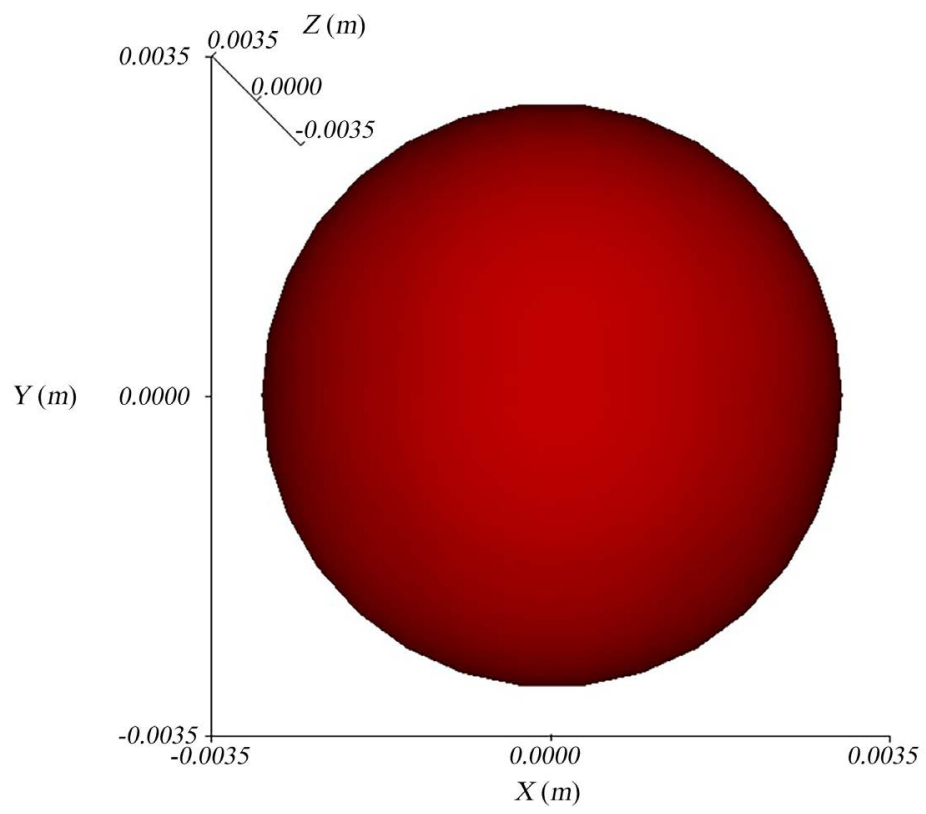

Figure 6. DEM particle.

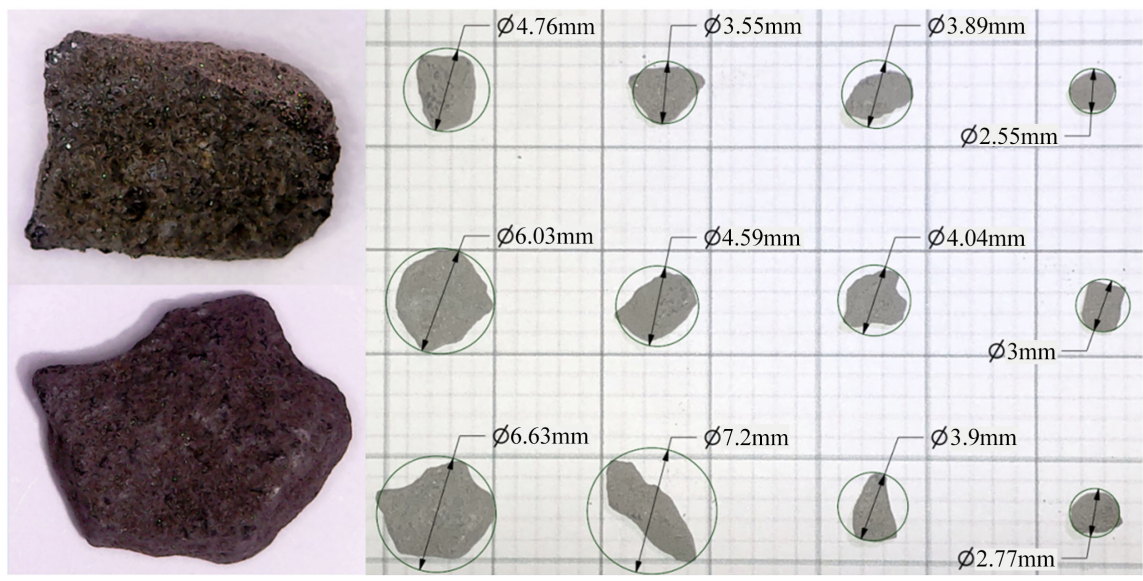

Figure 7. Shape and size of the iron pellet particles.

Device 1 with dimensions $0.23 \times 03 \times 0.175(\mathrm{~m})$, has a load flow of $7.5($ ton $/ \mathrm{h})$ and an adhesive distance of $0.0001(\mathrm{~m})$ these together with the parameters of the bulk material used from Table 2 gives us the following results for the repose and drawdown angles.

The second test device preserves two measurements of device 1 which are the height and width, the depth is the one that is affected by the change in dimensions reducing its size, with this the measurements of device 2 are $0.23 \times 0.3 \times$ $0.095(\mathrm{~m})$, the same as device 1 , the load flow is 7.5 (ton/h) and an adhesive distance of $0.0001(\mathrm{~m})$ resulting in the values in Table 3.

The third device with respect to the second maintains the dimension of width and depth; instead its height suffers an increase. Compared to device 1, the width remains constant, while the height increases and the depth decreases so that the dimensions of device 3 are $0.23 \times 0.59 \times 0.095(\mathrm{~m})$, in addition the flow 
of bulk material in the process it is 7.5 (ton/h) and for the test an adhesive distance of $0.0001(\mathrm{~m})$ will be used, with these parameters we obtain the results shown in Table 4.

\section{Results}

When there is a variation in the depth dimension of a test device as observed in test mechanism 2, in which the physical dimension in the depth of device 2 is decreased with respect to device 1 , it is observed in the shear angle both on the right and on the left as observed in Table 5, which generates a standard deviation around $2 \%$ in each case, so the drawdown angle is not very sensitive to the change in the depth dimension.

The height dimension is modified as it happens in device 3 in which with respect to mechanism 2 the width and depth measurement is kept constant, generating an increase in its height, this produces a variation in the drawdown angles as observed in Table 5, the value of the angle generated on the right has a decreased of $16 \%$ in the case of the left side, the value decreases by $13 \%$ with respect to the values obtained from device 2 .

Table 2. Device 1 adhesive distance: 0.0001 (m).

$\begin{array}{cc}\text { Time simulation } & 2: 58: 53 \\ \text { Particles quantity } & 5134 \text { partículas } \\ \text { Angle of repose right } & 35^{\circ} \\ \text { Angle of repose left } & 35^{\circ} \\ \text { Drawdown angleright } & 50^{\circ} \\ \text { Drawdown angle left } & 51^{\circ}\end{array}$

Table 3. Device 2 adhesive distance: 0.0001 (m).

\begin{tabular}{cc}
\hline Time simulation & $1: 41: 43$ \\
\hline Particles quantity & 2569 partículas \\
Angle of repose right & $33^{\circ}$ \\
Angle of repose left & $34^{\circ}$ \\
Drawdown angleright & $51^{\circ}$ \\
Drawdown angle left & $52^{\circ}$ \\
\hline
\end{tabular}

Table 4. Device 3 adhesive distance: 0.0001 (m).

\begin{tabular}{cc} 
Time simulation & $2: 55: 46$ \\
Particles quantity & 5057 partículas \\
Angle of repose right & $39^{\circ}$ \\
Angle of repose left & $40^{\circ}$ \\
Drawdown angleright & $43^{\circ}$ \\
Drawdown angle left & $45^{\circ}$ \\
\hline
\end{tabular}


Table 5. Drawdown angle.

\begin{tabular}{lll}
\hline & Right & Left \\
\hline Device 1 & $50^{\circ}$ & $51^{\circ}$ \\
Device 2 & $51^{\circ}$ & $52^{\circ}$ \\
Device 3 & $43^{\circ}$ & $45^{\circ}$ \\
\hline
\end{tabular}

In the case of the angles of repose, the value of the left side presents a decrease of $1^{\circ}$ as seen in Table 6 , for the case of the angle generated on the right side it is observed that it decreases by $2^{\circ}$ with respect to the value obtained from device 1 , with which a deviation of $6 \%$ is generated on the right side and $3 \%$ on the left sector.

In the values presented in Table 6, it is observed that the resting angles obtained in the test of device 3 suffer an increase with respect to the second, for the case of the angle that is formed to the right and to the left it is observed a growth of $18 \%$.

There is a decrease in the calculation time, with device 1 being the one that takes the longest to process to obtain the final results as presented in Table 7, this is due to the fact that this mechanism has a greater quantity of particles and longer fill time when testing starts.

When decreasing one of the dimensions of the test device, the calculation time decreases, on the contrary when the dimensions of the test mechanism are increased, the calculation time suffers an increase, this is observed since device 3 suffers an increase in the height with respect to device 2, which generates an increase in the processing time as can be seen in Table 7.

When reducing one of the dimensions of the test device, the calculation time decreases, on the contrary, when the dimensions of the test mechanism are increased, the calculation time suffers an increase, this occurs when device 3 suffers and increase in height with respect to device 2, which generates an increase in processing time as can be seen in Table 7.

Figure 8 shows a comparison between the drawdown angles of the right and left side obtained in the 3 devices.

Figure 9 shows a comparison of the angles of repose of the right and left side respectively, obtained from the tests carried out on the 3 devices.

It is observed that when modifying the value of the depth of the test device the variation of the results is of maximum order of $6 \%$ as observed in the comparison of device 1 and 2, on the contrary the results are very sensitive to the change in dimension of the height of the device, since the angle of repose on the left side presents a difference of $18 \%$ between the values obtained from devices 2 and 3 . The calculation times are directly proportional to the change in dimensions, since if there is a decrease in depth the processing time tends to decrease, if one of its dimensions increases the processing time calculation increases, one of the causes is that the quantity of particles varies proportionally to the dimensions of the device. 
Table 6. Repose angle.

\begin{tabular}{lcl}
\hline & Right & Left \\
\hline Device 1 & $35^{\circ}$ & $35^{\circ}$ \\
Device 2 & $33^{\circ}$ & $34^{\circ}$ \\
Device 3 & $39^{\circ}$ & $40^{\circ}$ \\
\hline
\end{tabular}

Table 7. Simulation calculation time for the different devices.

\begin{tabular}{ll}
\hline & Time \\
\hline Device 1 & $2: 58: 53$ \\
Device 2 & $1: 41: 34$ \\
Device 3 & $2: 55: 46$ \\
\hline
\end{tabular}

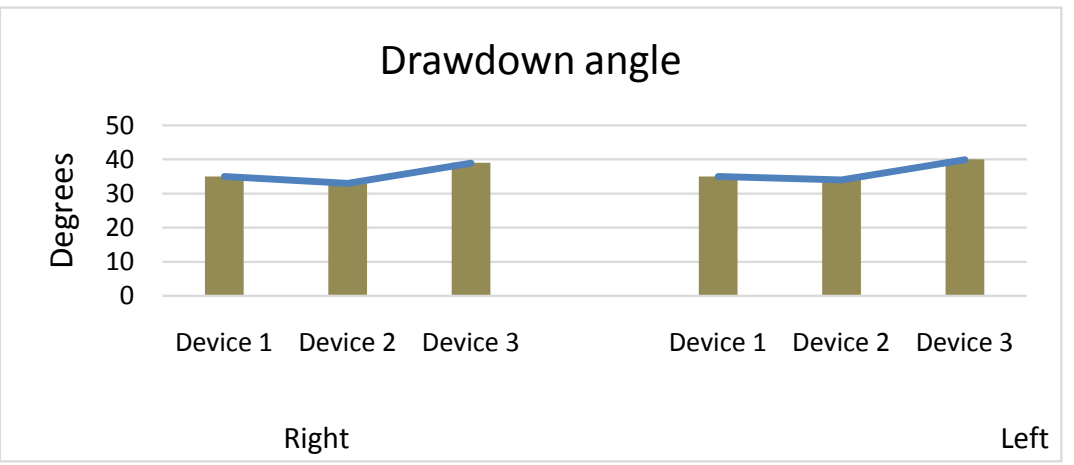

Figure 8. Right Drawdown angle comparison.

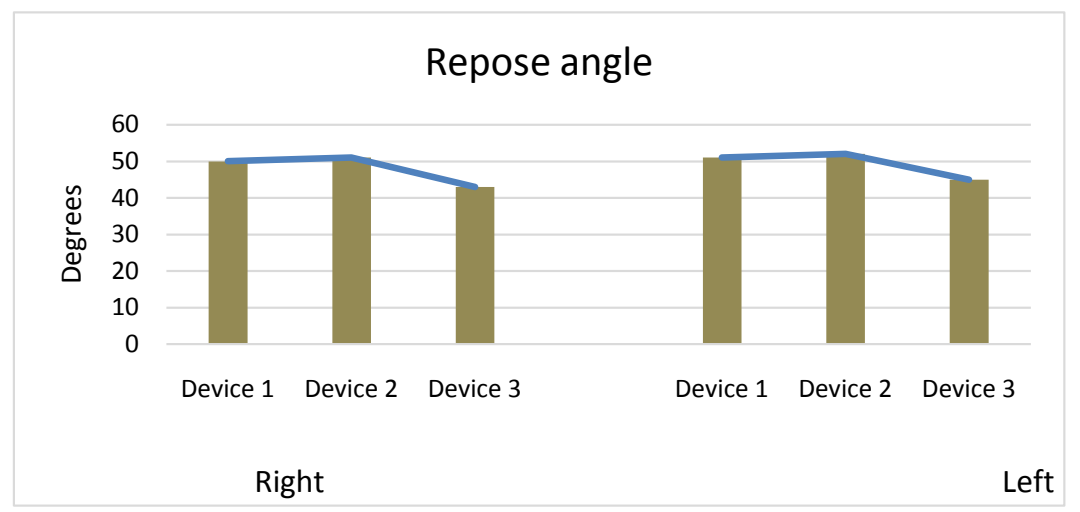

Figure 9. Right angle of repose comparison.

\section{Conclusions}

From the results obtained, it can be established that calibration is an important factor to be able to obtain reliable and realistic results of simulations using the discrete elements method of solid granular material composed of iron pellets for the simulation of industrial processes that process this type of material since it allows to define precisely the parameters that must be entered as input variables in them, that is why defining the appropriate dimensions will allow the calibra- 
tion values to be defined more quickly and precisely.

A dimensional variable that significantly affects the results of the tests is the height of the device, so special care must be taken in the construction of test devices with an adequate height to be able to calibrate the parameters correctly. In this sense, the dimensions proposed for the construction of the test devices must be in an intermediate range of the dimensions studied.

Another significant aspect is the calculation times experienced by the simulations of the test carried out, where the choice of intermediate sizes of the studied devices will allow to run a greater number of simulations to establish statistically reliable significant figures. The substantial savings in the calibration processes will reduce the time involved in the calibration processes, ensuring simulation results that are more adjusted to the real phenomena of processes and equipment that handle solid granular iron pellet material.

It should be noted that the granulometry used in the study must be respected so that the results of the DEM simulations adhere to the phenomenology that the particles experience in their real performance as well as the real geometric shape of the particle. In this study, the equivalent volumes of the sizes of the real particles were used to approximate their behavior using a spherical shape. The changes that occur when using morphology similar to the real one will vary according to the number of sides and different volumetric dimensions of them. It is to be expected that using granulometries in the ranges mentioned and with geometric shapes identical to the real particles, results are obtained that are adjusted to the phenomena that actually occur.

Future research should be focused on conducting tests with physical devices for experimental validation and with geometric morphology of the real particle. Another research objective is to be able to determine the differences that occur in the experimental results due to the roughness of the surfaces and their effect on the static and dynamic friction coefficients of the particles.

From the above, it is a valuable contribution to the research to be able to determine with small modifications in the design of the values of the device of coefficients of friction and restitution with a single test procedure, in this case, it is necessary to investigate the governing and binding equations that describe what the particles experience during the granular material calibration testing process.

Obtaining reliable results of calibration values for DEM simulations will allow elucidating many of the phenomena that occur during the processes associated with the transport and processing of iron pellet materials with realistic and reliable simulations. The use of the methodology of discrete elements and its ability to couple other methodologies such as the elements and finite volumes will allow an important development in the mining industry, not only to verify and establish levels of processes but also in the effects that impact the useful life of assets by improving their maintenance strategies with a binding approach of condition monitoring techniques 


\section{Acknowledgements}

The authors gratefully acknowledge to faculty of engineering of the University of La Serena, "FIULS 2030" code 18ENI2-104235. Further, the authors appreciate the thorough and detailed comments of the anonymous specialists who reviewed this article.

\section{Conflicts of Interest}

The authors declare no conflicts of interest regarding the publication of this paper.

\section{References}

[1] Cundall, P.A. and Strack, O.D. (1979) A Discrete Numerical Model for Granular Assemblies. Geotechnique, 29, 47-65. https://doi.org/10.1680/geot.1979.29.1.47

[2] Coetzee, C.J. and Els, D.N.J. (2009) Calibration of Granular Material Parameters for DEM Modelling and Numerical Verification by Blade-Granular Material Interaction. Journal of Terramechanics, 46, 15-26. https://doi.org/10.1016/j.jterra.2008.12.004

[3] Gustafsson, G., Häggblad, H.Å., Jonsén, P. and Marklund, P. (2013) Determination of Bulk Properties and Fracture Data for Iron Ore Pellets Using Instrumented Confined Compression Experiments. Powder Technology, 241, 19-27. https://doi.org/10.1016/j.powtec.2013.02.030

[4] Qi, J., Li, K.C., Jiang, H., Zhou, Q. and Yang, L. (2015) GPU-Accelerated DEM Implementation with CUDA. International Journal of Computational Science and Engineering, 11, 330-337. https://doi.org/10.1504/IJCSE.2015.072653

[5] Peters, B. (2013) The Extended Discrete Element Method (XDEM) for Multi-Physics Applications. Scholarly Journal of Engineering Research, 2, 1-20.

[6] Zhao, J. and Shan, T. (2013) Coupled CFD-DEM Simulation of Fluid-Particle Interaction in Geomechanics. Powder Technology, 239, 248-258.

https://doi.org/10.1016/j.powtec.2013.02.003

[7] Ng, A.H.M., Chang, H.C., Ge, L., Rizos, C. and Omura, M. (2009) Assessment of Radar Interferometry Performance for Ground Subsidence Monitoring Due to Underground Mining. Earth, Planets and Space, 61, 733-745. https://doi.org/10.1186/BF03353180

[8] Xu, N., Zhang, J., Tian, H., Mei, G. and Ge, Q. (2016) Discrete Element Modeling of Strata and surface Movement Induced by Mining under Open-Pit Final Slope. International Journal of Rock Mechanics and Mining Sciences, 88, 61-76. https://doi.org/10.1016/j.ijrmms.2016.07.006

[9] Jylhä, J. (2018) CFD-DEM Simulation of Two-Phase Flows in the Flash Smelting Settler.

[10] Lei, X., Liao, Y. and Liao, Q. (2016) Simulation of Seed Motion in Seed Feeding Device with DEM-CFD Coupling Approach for Rapeseed and Wheat. Computers and Electronics in Agriculture, 131, 29-39. https://doi.org/10.1016/j.compag.2016.11.006

[11] Suzzi, D., Toschkoff, G., Radl, S., Machold, D., Fraser, S.D., Glasser, B.J. and Khinast, J.G. (2012) DEM Simulation of Continuous Tablet Coating: Effects of Tablet Shape and Fill Level on Inter-Tablet Coating Variability. Chemical Engineering Science, 69, 107-121. https://doi.org/10.1016/j.ces.2011.10.009 
[12] Richter, C., Rößler, T., Kunze, G., Katterfeld, A. and Will, F. (2020) Development of a Standard Calibration Procedure for the DEM Parameters of Cohesionless Bulk Materials Part II: Efficient Optimization-Based Calibration. Powder Technology, 360, 967-976. https://doi.org/10.1016/j.powtec.2019.10.052

[13] Gustafsson, G., Häggblad, H.Å. and Jonsén, P. (2013) Multi-Particle Finite Element Modelling of the Compression of Iron Ore Pellets with Statistically Distributed Geometric and Material Data. Powder Technology, 239, 231-238. https://doi.org/10.1016/j.powtec.2013.02.005

[14] Rackl, M. and Hanley, K.J. (2017) A Methodical Calibration Procedure for Discrete Element Models. Powder Technology, 307, 73-83.

https://doi.org/10.1016/j.powtec.2016.11.048

[15] Roessler, T. and Katterfeld, A. (2016) Scalability of Angle of Repose Tests for the Calibration of DEM Parameters. 12 th International Conference on Bulk Materials Storage, Handling and Transportation (ICBMH 2016), Darwin, 11-14 July 2016, 201.

[16] Do, H.Q., Aragón, A.M. and Schott, D.L. (2018) A Calibration Framework for Discrete Element Model Parameters Using Genetic Algorithms. Advanced Powder Technology, 29, 1393-1403. https://doi.org/10.1016/j.apt.2018.03.001

[17] Yoon, J. (2007) Application of Experimental Design and Optimization to PFC Model Calibration in Uniaxial Compression Simulation. International Journal of Rock Mechanics and Mining Sciences, 44, 871-889.

https://doi.org/10.1016/j.ijrmms.2007.01.004

[18] Benvenuti, L., Kloss, C. and Pirker, S. (2016) Identification of DEM Simulation Parameters by Artificial Neural Networks and Bulk Experiments. Powder Technology, 291, 456-465. https://doi.org/10.1016/j.powtec.2016.01.003

[19] Luding, S. (2008) Introduction to Discrete Element Methods: Basic of Contact Force Models and How to Perform the Micro-Macro Transition to Continuum Theory. European Journal of Environmental and Civil Engineering, 12, 785-826. https://doi.org/10.1080/19648189.2008.9693050

[20] Zhu, H.P., Zhou, Z.Y., Yang, R.Y. and Yu, A.B. (2007) Discrete Particle Simulation of Particulate Systems: Theoretical Developments. Chemical Engineering Science, 62, 3378-3396. https://doi.org/10.1016/j.ces.2006.12.089

[21] González, C.A.L. (2012) Advances in the Development of the Discrete Element Method for Excavation Processes. Doctoral Dissertation, Universitat Politècnica de Catalunya (UPC).

[22] Oñate, E., Labra, C., Zárate, F., Rojek, J. and Miquel, J. (2005) Avances en el desarrollo de los Métodos de Elementos Discretos y de Elementos Finitos para el análisis de problemas de fractura. Anales de Mecánica de la Fractura, 22, 27-34.

[23] Taylor, L.M. and Preece, D.S. (1992) Simulation of Blasting Induced Rock Motion Using Spherical Element Models. Engineering Computations, 9, 243-252. https://doi.org/10.1108/eb023863

[24] Katterfeld, A., Coetzee, C., Donohue, T., Fottner, A.J., Grima, A., Gomez, A.R., Schott, D., et al. (2019) Calibration of DEM Parameters for Cohesionless Bulk Materials under Rapid Flow Conditions and Low Consolidation.

[25] Rackl, M., Grötsch, F.E., Rusch, M. and Fottner, J. (2017) Qualitative and Quantitative Assessment of 3D-Scanned Bulk Solid Heap Data. Powder Technology, 321, 105-118. https://doi.org/10.1016/j.powtec.2017.08.009

[26] Barrios, G.K., de Carvalho, R.M., Kwade, A. and Tavares, L.M. (2013) Contact Parameter Estimation for DEM Simulation of Iron Ore Pellet Handling. Powder 
Technology, 248, 84-93. https://doi.org/10.1016/j.powtec.2013.01.063

[27] Guya, S.R. (2018) Calibration of Discrete Element Modelling Parameters for Bulk Materials Handling Applications (Doctoral Dissertation).

[28] Chen, G., Lodewijks, G. and Schott, D.L. (2018) Numerical Prediction on Abrasive Wear Reduction of Bulk Solids Handling Equipment Using Bionic Design. Particulate Science and Technology, 37, 964-973. https://doi.org/10.1080/02726351.2018.1480547

[29] Schott, D., Vreeburg, W., Molhoek, C. and Lodewijks, G. (2016) Granular Flow to a Blast Iron Ore Furnace: Influence of Particle Size Distribution on Segregation of a Mixture. In: Traffic and Granular Flow'15, Springer, Cham, 621-628. https://doi.org/10.1007/978-3-319-33482-0_78

[30] Coetzee, C.J. (2016) Calibration of the Discrete Element Method and the Effect of Particle Shape. Powder Technology, 297, 50-70.

https://doi.org/10.1016/j.powtec.2016.04.003 